\title{
Timing of food intake predicts weight loss effectiveness
}

\author{
Prof Marta Garaulet ${ }^{1}$, Purificación Gómez-Abellán, $\mathrm{PhD}^{1}$, Juan J Alburquerque-Béjar, \\ PhD1, Yu-Chi Lee, PhD ${ }^{2}$, Prof Jose M Ordovás ${ }^{2,3,4}$, and Prof. Frank AJL Scheer ${ }^{5,6}$ \\ ${ }^{1}$ Department of Physiology. Faculty of Biology. University of Murcia. Spain
}

${ }^{2}$ Nutrition and Genomics Laboratory. Jean Mayer US Department of Agriculture Human Nutrition Research Center on Aging, at Tufts University, Boston, MA

${ }^{3}$ Department of Epidemiology, Centro Nacional Investigaciones Cardiovasculares (CNIC), Madrid, Spain

${ }^{4}$ Instituto Madrileño de Estudios Avanzados en Alimentación (IMDEA-FOOD), Madrid, Spain

${ }^{5}$ Medical Chronobiology Program, Division of Sleep Medicine, Brigham and Women's Hospital

${ }^{6}$ Division of Sleep Medicine, Harvard Medical School, Boston, MA

\section{Abstract}

Background-There is emerging literature demonstrating a relationship between the timing of feeding and weight regulation in animals. However, whether the timing of food intake influences the success of a weight-loss diet in humans is unknown.

Objective-To evaluate the role of food-timing in weight-loss effectiveness in a sample of 420 individuals who followed a 20 -week weight-loss treatment.

Methods-Participants (49.5\% females; age [mean+/-SD]: $42 \pm 11$ years; BMI: $31.4 \pm 5.4 \mathrm{~kg} / \mathrm{m}^{2}$ ) were grouped in early-eaters and late-eaters, according to the timing of the main meal (lunch in this Mediterranean population). $51 \%$ of the subjects were early-eaters and $49 \%$ were late-eaters (lunch time before and after 3:00 PM, respectively), energy intake and expenditure, appetite hormones, CLOCK genotype, sleep duration and chronotype were studied.

Results-Late lunch eaters lost less weight and displayed a slower weight-loss rate during the 20 weeks of treatment than early-eaters $(P=0.002)$. Surprisingly, energy intake, dietary composition, estimated energy expenditure, appetite hormones and sleep duration was similar between both groups. Nevertheless, late-eaters were more evening-types, had less energetic breakfasts, and skipped breakfast more frequently that early-eaters $(P<0.05)$. CLOCK rs4580704 SNP associated with the timing of the main meal $(P=0.015)$ with a higher frequency of minor allele $(C)$ carriers among the late-eaters $(P=0.041)$. Neither sleep duration, nor $C L O C K$ SNPs or Morning/Evening chronotype was independently associated with weight-loss $(P>0.05)$.

Conclusions-Eating late may influence the success of weight-loss therapy. Novel therapeutic strategies should incorporate not only the caloric intake and macronutrient distribution-as is classically done-but also the timing of food.

Corresponding author: Marta Garaulet. Department of Physiology. Faculty of Biology. University of Murcia. Campus de Espinardo, s/ n. 30100. Murcia, Spain. Phone: +34868 88 39 30. Fax: +34 8688839 63. garaulet@um.es.

Conflict of interest: M.G., P.G.A., J.J.A.B., Y.C.L., J.M.O. and F.A.J.L.S. have no conflict of interest.

Authors' contributions to manuscript: M.G., F.A.J.L.S. designed research; M.G., P.G.A., J.J.A.B., Y.C.L., J.M.O. conducted research; M.G., F.A.J.L.S. analyzed data; M.G., F.A.J.L.S. wrote the paper; M.G. had primary responsibility for final content. All authors read and approved the final manuscript. 


\section{Keywords}

Timing of food intake; obesity; weight loss; dietary treatment; circadian

\section{Introduction}

Many factors have been recognized to influence the success of a dietary intervention in obesity (1). Indeed, obesity is a heterogeneous condition and individual responses to standardized protocols targeting weight loss are highly variable (2). In real life settings such as obesity treatment programs, physiological and psychological factors, some of which may carry a strong genetic influence, interact with environmental factors in a complex manner (3-5). However, the current therapeutic strategies are mostly focused on the imbalance between energy expenditure and caloric intake. Recent studies link energy regulation to the circadian clock at the behavioural, physiological, and molecular levels (6-9), emphasizing that the timing of food intake itself may play a significant role in weight regulation (10).

In this regards, there is emerging literature in animals demonstrating a relationship between the timing of feeding and weight regulation (11). Rhythmic feeding appears to be the major synchronizer for peripheral oscillators $(10,11)$. Thus, unusual feeding time can produce a disruption of the circadian system by inducing internal desynchronisation trough decoupling of peripheral oscillators from the central clock, the suprachiasmatic nucleus (SCN) (12). This differential synchronization induced by abnormal feeding habits might produce unhealthy consequences also in humans (13).

Furthermore, one of the most influential discoveries relevant for this area of research in the last years is the presence of an active circadian clock in adipose tissue (14). New data suggest that there is a temporal component in the regulation of adipose tissue functions (15). In fact, studies performed by microarrays have shown that a substantial percentage of active genes expressed in adipose tissue in both humans and animal models follow a daily rhythmic pattern $(16,17)$. Thus, a specific temporal order in the daily patterns of these genes appears to be crucial for adipose tissue to exclusively either accumulate fat or to mobilize fat at the proper time, a phenomenon known as temporal compartmentalization (18). Taking into account that feeding is the source of energy for adipose tissue, the time of feeding, particularly for high energy content meals, may be decisive and changes in this timing could have metabolic consequences for the development of obesity and perhaps for weight loss.

Over the past two decades, biochemical, genetic, and molecular studies have been making substantial advances towards the elucidation of the molecular bases of rhythmicity in biology. From the genetic epidemiology point of view, the study of single nucleotide polymorphisms (SNPs) is contributing to the identification of the genetic background of chronotypes (morningness or eveningness), sleep alterations or seasonal mood disorders (19). What is more, different studies have already related clock genes SNPs with obesity (5) and weight loss (20). Nevertheless, whether the timing of food intake is related to clock SNPs and more importantly, if the timing of food intake influences the success of a weightloss diet in humans is still unknown. Given all the above mentioned, the goal of this study was to evaluate the role of food timing in weight loss in a sample of 420 participants following a behavioural treatment for obesity based on a Mediterranean diet. Behavioural, physiological, and genetic aspects that could influence differences in weight loss response were also considered. 


\section{Subjects and Methods}

\section{Participants and procedures}

Between 2007 and 2008, an initial sample of 510 subjects (49.5\% women) (age [mean+/ $-\mathrm{SD}$ ]: $42 \pm 11$ years; BMI: $31.4 \pm 5.4 \mathrm{~kg} / \mathrm{m}^{2}$ ) were voluntarily attending five nutrition clinics in the southeast of Spain for dietetic and behavioural treatment to lose weight based on the principle of the Mediterranean diet and behavioural and cognitive techniques (Método Garaulet( $)$ which was described previously (1). Patients attended an outpatient clinic specializing in obesity treatment for the first time or were expatients who had not participated in the program for at least two years. All subjects came from the city of Murcia, located in the southeast of Spain on the coast of the Mediterranean Sea. 18\% of the initial volunteer subjects were excluded because they were subjects on a special diet, under treatment with weight loss medication, or they had a diagnosis of diabetes mellitus, chronic renal failure, hepatic diseases, or cancer. Finally, a total of 420 overweight and obese subjects participated in the study. Dietary intake was assessed in all participants before the beginning of the treatment and results were monitored during a 20 -week treatment period. All procedures were in accordance with good clinical practice. Patient data were codified to guarantee anonymity.

\section{Ethics}

Written informed consent was obtained before subjects were accepted and was performed in accordance with the Helsinki Declaration of Human Studies of 1975 as revised in 1983 and approved by the Ethical Committee of the University of Murcia.

\section{Intervention}

The structure of the program has been described elsewhere in detail (21). Subjects attended 60-minute therapy sessions once per week in groups of 10. Duration of the programme was variable depending on the weight loss goal, although it lasted approximately 5 months in most of the patients studied. Once they achieved their weight loss goals, they followed a 5month maintenance period. During maintenance, meetings were held every 2 weeks initially and monthly afterward. Weight loss was recorded weekly during the first part of the treatment (the weight loss part) in order to study the weight loss evolution. Throughout the whole program, sessions were conducted by a food and nutritional professional. Treatment was based on four main components: (1) dietetic treatment, based in the principles of the Mediterranean diet, in which the distribution of macronutrient components followed the recommendations of the Spanish Society of Community Nutrition (22); (2) nutritional education; (3) moderate physical activity intervention; and (4) cognitive-behavioral techniques, including stimulus control, self-monitoring, positive reinforcement, preventing relapse and cognitive restructuring, following a structured protocol (23). A complete description of the intervention and the effectiveness of the intervention can be found in Corbalán et al. (2009) (1). Patients received recommendations about the number of portions that they should eat from each group of food. However, no advice was given to the patients with regard to the food timing and daily energy intake distribution during the treatment.

\section{Obesity and Metabolic Syndrome Parameters}

Each participant was weighed while barefoot, wearing light clothes, on a digital scale that measured to the nearest $0.1 \mathrm{~kg}$, at the same time of the day (in the evening), once per week before and throughout the treatment period. Height was measured using a Harpenden digital stadiometer (rank 0.7-2.05). Each participant was positioned upright, and relaxed, with head on the Frankfurt plane. BMI was calculated as weight $(\mathrm{kg}) /$ height $(\mathrm{m})^{2}$. Total body fat was measured with bioelectrical impedance using TANITA TBF-300 (TANITA Corporation of 
America) equipment. Body fat distribution was assessed by anthropometric measures, including waist circumference, at the level of the umbilicus, and hip circumference, with the widest circumference over the greater trochanters (24). All measurements were made with a flexible and inextensible measuring tape.

Glucose, cholesterol (C), triglycerides (TG), lipoproteins concentrations of plasma were determined by automated chemical analysis (IL ILAB 600 Chemistry Analyzer of Instrumentation Laboratory). HDL-C was measured after precipitation of apoB-containing lipoproteins with dextran sulfate and magnesium (27).. LDL-C was calculated as total cholesterol (TC) - (HDL-C + VLDL-C ), when the TG was $<4.52 \mathrm{mmol} / \mathrm{L}$ (28). Insulin was determined through a solid-phase, 2 -site chemiluminescent immunometric assay (IMMULITE 2000 Insulin). The homeostasis model assessment (HOMA) index of insulin resistance was calculated with the accepted formula: HOMA $=$ fasting glucose $(\mathrm{mmol} / \mathrm{l}) \mathrm{x}$ fasting insulin $(\mathrm{mIU} / \mathrm{l}) / 22.5$.

Blood pressure was measured with participants seated with the arm resting on a table. Blood pressure readings were measured in $\mathrm{mm} \mathrm{Hg}$. We used the Joint Interim Statement of the International Diabetes Federation Task Force on Epidemiology and Prevention; National Heart, Lung, and Blood Institute; American Heart Association; World Heart Federation; International Atherosclerosis Society; and International Association for the Study of Obesity guidelines to classify patients for metabolic syndrome (MetS), (25)

\section{Energy intake before and during treatment}

To evaluate habitual dietary intake before the treatment, intake was determined by the "24-h dietary recall", including 24-h recalls of food intake from all days of the week in the total population. Dietary intake during the treatment was assessed by the "7-day dietary record" measuring and weighting food daily. Subjects recorded their dietary intakes for 7 days, every week during the whole intervention. Patients also recorded the time of day that each meal (e.g., breakfast, lunch, and dinner) was eaten. After excluding atypical weeks due to vacations, sickness etc. we randomly selected one week from the total treatment in each patient to analyze data in order to cover the 20 weeks of treatment and to obtain an overall picture of the dietary intake along the whole weigh loss period. Importantly, patients indicated in each weekly notebook whether or not this was a usual week, and after double checking by the nutritionist, a representative week was chosen for the study.

Total energy intake and macronutrient composition were analyzed with the nutritional evaluation software program Grunumur (26), on the basis of Spanish food composition tables $(27,28)$.

\section{Energy expenditure}

Total energy expenditure was calculated by multiplying each individual's basal metabolic rate (BMR) with the individual activity level as assessed by METs (metabolic equivalents). BMR was estimated by the Harris-Benedict equation. METs were calculated by use of the International Physical Activity Questionnaire (IPAQ) that was administered with the help of a nutritionist and assessed physical activity over the last 7 days. The IPAQ was developed for adults between 18-65 years, assessing the different domains of physical activity (work, transport, house and garden and leisure time). A total activity score reflecting intensity and time was calculated as METs expressed in minutes per week for the four domains combined. The IPAQ instrument has been validated internationally and in a Spanish population, in which good correlation with accelerometer data was obtained $(29,30)$. 


\section{Morningness/Eveningness Questionnaire}

Subjects completed the morningness-eveningness questionnaire (MEQ) 19-item scale of Horne and Ostberg, 1976 (31). Morningness-eveningness typology is a way to characterize subjects depending on individual differences of wake/sleep patterns and the time of day people feel or perform best. Some people are night "owls" and like to stay up late at night and sleep late in the morning (Evening type), while others are early birds and prefer to go to bed early and arise with the break of dawn (Morning types). The majority of people is in between these two extremes and is categorized as "Neutral types" (32). Evening types were those scoring under 53 and morning types were those scoring above 64. All subjects within the range of 53 to 64 were classified as Neutral type (32).

\section{Sleep Duration}

Habitual sleep duration was estimated by a questionnaire. 'During week days: How many hours (and minutes) do you usually sleep?' and; 'During weekend days: How many hours (and minutes) do you usually sleep?'. A total weekly sleep score was calculated as: ((min Weekdays x 5) + (min Weekend days x 2))/7 (3).

\section{Appetite hormones: Leptin and Ghrelin}

Fasting blood samples, collected at $8 \mathrm{AM}$ were centrifuged at $4^{\circ} \mathrm{C}$, and the plasma was stored at $-70^{\circ} \mathrm{C}$ for subsequent analysis. Plasma leptin and ghrelin samples were measured by radioimmunoassay (Linco Research, St. Charles, MO). All samples for leptin and ghrelin were run in duplicate.

\section{DNA isolation and clock genotyping}

DNA was isolated from blood samples using routine DNA isolation sets (Qiagen, Valencia, CA, USA). We performed genotyping of CLOCK gene polymorphisms using a TaqMan assay with allele-specific probes on the ABI Prism 7900HT Sequence Detection System (Applied Biosystems, Foster City, CA, USA). We selected tag single-nucleotide polymorphisms (tSNPs) as effective proxies for untyped SNPs in strong linkage disequilibrium (LD) by using the Tagger23 based on HapMap Caucasian European Utah data (www.hapmap.org) with a minimum minor allele frequency (MAF) 0.10 and a minimum $\mathrm{r}^{2}$ of 0.8 . Different SNPs of CLOCK previously related with obesity or metabolic syndrome rs3749474, rs1801260, rs4864548 and rs4580704 were selected $(33,34,35)$.

\section{Statistics}

Firstly, subjects were dichotomized in early and late eaters for breakfast, lunch and dinner using the median values of the population as the cut-off point. Differences between early and late eaters in weight loss were analyzed by ANOVA. To study weight loss evolution during 20 weeks of treatment a repeated-measures analysis of variance (ANOVA) was used. Further ANOVA analyses were only performed for lunch eaters because this was the only meal timing associated with weight loss. Chi-square tests were also used to test differences in percentages between late and early eaters. A dominant model was applied in every CLOCK SNP as previously described $(33,34,35)$. ANOVA and partial correlation analyses were also used to test independent associations between sleep duration, chronotype and genotype and weight loss. We adjusted analyses for sex, age, nutritional clinic, and BMI. Statistical analyses were performed using SPSS 15.0 software (SPSS). A two-tailed P-value of $<0.05$ was considered statistically significant. 


\section{Results}

Our results indicate that late lunch eaters lost significantly less weight than early eaters (Figure 1). As shown in Figure 1, the weight loss pattern during the 20 weeks of treatment also differed between late and early eaters in a way that late eaters displayed a slower rate of weight loss starting after the $5^{\text {th }}$ week of treatment, a difference that was maintained during the remaining intervention period $(P=0.002$; Figure 1). Differences were significant for total weight loss $(\mathrm{kg})$, percentage of weight loss from initial weight, and weekly weight loss rate (Table 1). However, no differences were found in weight loss dependent on the timing of breakfast or dinner (see Table 1).

Table 2 represents the initial characteristics of the subjects studied depending on the median value of meal intake (before and after $3 \mathrm{PM}$ ). Because no interaction was found with gender for the main outcome (weight loss), data from men and women were pooled together. No significant differences were found in age, gender distribution, obesity-related variables and metabolic syndrome characteristics between the early and late lunch eaters except for HOMA, an index of insulin resistance, which was significantly higher in the late eaters.

Habitual energy intake, total energy expenditure and physical activity were also similar between the late and early eaters. Moreover, no significant differences were found between the two groups studied in appetite hormones and sleep duration. Nevertheless, late eaters were more evening types (lower MEQ score) than early eaters as assessed by the morningness-eveningness questionnaire $(P=0.032)$.

Regarding the genetic background from the different SNPs (Table 2), for CLOCK rs 4580704 , the minor allele $(\mathrm{G})$, that has been previously associated with obesity (33) was significantly more frequent among late eaters than in early eaters $(P=0.015)$. Furthermore, the minor allele carriers $[\mathrm{G}]$ had lunch significantly later than major allele carriers (Mean \pm SD: $14: 44 \pm 00: 33$ vs. $14: 36 \pm 00: 28$, respectively; $P=0.001$ ). No significant associations were found between meal timing and the other CLOCK SNPs studied.

The characteristics of the diet during the weight loss intervention between late and early eaters are shown in Table 3. Surprisingly, total daily energy intake was similar between both groups. Moreover, no significant differences were found for dietary composition during the weight loss treatment.

In this Mediterranean population, lunch constituted the meal with the highest energy content, $40 \%$ of total daily energy intake (see Table 3). It is important to note that no significant differences were found between late and early eaters for lunch and dinner energy intake distribution. However, late eaters had less energetic breakfasts (\% of daily energy intake; $P=0.031)$, and skipped breakfast more frequently that early eaters (6.6\% versus $2.6 \% ; P=0.039$ ). With respect to the macronutrients distribution in lunch, no significant differences were found, apart from a lower protein intake among late eaters (Table 3).

Of note, the timing of lunch intake was significantly and positively correlated with the timing of dinner $(\mathrm{r}=0.171 ; P=0.0004)$ in a way that late eaters for lunch, were also late eaters for dinner. However no significant correlations were found between the timing of lunch and that of breakfast $(\mathrm{r}=0.042 ; P=0.393)$.

One notable outcome was that sleep duration, CLOCK SNP and Morning/Evening chronotype, were not independently associated with weight loss $(P>0.05$, ANOVA; Table 4). There was also no significant correlation between weight loss and chronotype within either the early eaters $(\mathrm{r}=0.04 ; P=0.57)$ or the late eaters $(\mathrm{r}=-0.02 ; P=0.89)$, separately, 
suggesting that differences in weight loss between both groups were not due to a concurrent predisposition to a chronotype and the timing of food.

\section{Discussion}

This is the first prospective longitudinal study to show that timing of food intake relates with weigh loss effectiveness in humans. In 420 overweight/obese patients undergoing a 20-week weight-loss diet, those who ate their main meal late lost significantly less weight than early eaters. This difference in weight loss success was not explained by differences in caloric intake, macronutrient distribution, or energy expenditure. Timing of food intake was associated with genetic variance in $C L O C K$, morningness-eveningness, lunch protein intake, and HOMA. As far as we are aware, no previous studies have investigated the relationship between the main meal timing and the long term effectiveness of a weight loss dietary treatment.

Despite the lack of such reports, there is an increasing number of publications showing the effect of meal timing on obesity. Of interest are the animal studies indicating that reversal of the feeding rhythm causes adverse metabolic effects and that correcting the timing of food intake can even prevent metabolic disturbances in animals on a high-fat diet $(10,11,37)$. In humans, it has been recently published by Jakubowicz et al. (2012) how a high carbohydrate and protein breakfast may prevent weight regain during a maintenance period by reducing diet-induced compensatory changes in hunger, cravings and ghrelin suppression (36). Moreover, in 1987 Sensi and Capani (39) published an elegant study in 19 subjects in which they investigated potential differences in weight loss with differences in food timing (one only meal at 10:00 AM or at 18:00 PM). However, weight loss did not vary between morning and afternoon eaters, though they found differences in lipids and carbohydrates oxidation with meal timing. An important aspect to consider is that the intervention was performed only during 18 days, and this short period of treatment seems to be insufficient to observe significant weight changes if we consider that in the current work differences with meal timing became apparent only at the $5^{\text {th }}$ week of intervention (after 35 days).

One of the most surprising findings in our study was that there were no significant differences in total energy intake or energy expenditure between late and early eaters despite the disparity in weight loss magnitude. Previous studies have had similar surprising outcomes. Arble et al. (10) showed that nocturnal mice fed a high-fat diet during the day gained significantly more weight than mice fed at night, in spite of both groups consuming equivalent amounts of calories and exhibited similar levels of locomotor activity. Several other experiments have been performed in Zucker rats $(40)$ and mice $(11,38,40)$ with similar conclusions, indicating that the timing of food intake was a critical factor mediating increased weight gain independently of energy intake. In humans, the studies on the association between food timing and obesity have been focused particularly on shift workers. The majority of evidence indicates that although shifts workers are more prone to obesity than day workers, total energy intake over 24 hours does not vary between day and shift workers or, between different work shift (41). We estimated energy expenditure using the Harris-Benedict formula and METs. Even though we found no differences in either energy intake or expenditure between the late and early eaters, we cannot exclude the possibility that the groups had differences in resting energy expenditure not predicted by their weight, height, gender and age. Moreover, one limitation of the current work is that the Harris-Benedict equation is not very accurate to assess energy expenditure, especially during weight loss.

Additionally, human studies on non-breakfast eaters and night-eating syndrome patients are also consistent with the concept that timing of food intake is a determining factor in weight 
gain $(42,43)$. Of note, it has been published recently how high carbohydrate and protein breakfast may prevent weight, possibly in part by changes in the recently demonstrated circadian control of hunger and appetite $(36,53)$. Along these lines, our results show that late eaters had a significantly lower percentage of their total daily energy intake during breakfast, and skipped breakfast more frequently than early eaters an effect that could be contributing to the differences in weight loss with lunch timing.

Although the mechanisms linking meal timing and weight loss is unknown, satiety hormones may be involved (10). Changes in the levels of circulating satiety hormones, such as leptin or ghrelin, by circadian misalignment could influence energy intake and expenditure $(42,43)$. However, in the current study no significant differences were found between early and late eaters in both appetite hormones. These results were not surprising considering that no differences in total daily energy intake were found either.

Multiple studies have shown the association between short sleep duration, increased risk for obesity (44-46) and impaired weight loss (3). However, our data indicated no overall differences in sleep duration between late and early eaters. This shows similarity with the observation in nocturnal mammals in which daytime feeding, that results in obesity, was not associated with decreased sleep duration (10). Moreover, a previous human study showed that the caloric consumption after 8 PM was associated with increased BMI independently of sleep timing and duration (47).

Genetics is another factor to consider in the association between meal timing and weight loss. Indeed, in the current population, rs4580704-a C/G variation in the CLOCK gene (intronic) on human chromosome 4-was related to the time of lunch, and a higher frequency of minor allele carriers was present among late eaters. This genetic variation has been previously related to individual susceptibility to obesity and metabolic risk $(33,34)$. Of note, this variant has been also associated with energy intake; with minor allele carriers showing higher energy intake (34). The circadian system must continuously adapt to and synchronize our physiology with the environment (48). Genetic variance in clock genes may be important in meal timing, possibly in part by changes in the recently demonstrated circadian control of hunger and appetite (53). Indeed, this is the first study to report the association of clock genetic variations with the timing of food intake. Earlier studies have identified allelic variants in $L E P$ and $L E P R$ genes that significantly influence the energy intake distribution across meals, but not the time of meals (48).

Another possible explanation for our result is that late eaters were more evening types as determined by the morningness-eveningness questionnaire. Previous studies indicate that evening types have more propensity to put on weight and less ability to lose it (4) and a delayed phase of the circadian rhythmicity has been associated with metabolic alterations and obesity $(49,50)$.

In theory, differences in sleep duration, chronotype and genetic variants could have explained the observed differences in weight loss between late and early eaters. Nevertheless, none of these factors were independently associated with weight loss. Although we do not discard the possibility that these effects may be additive as previously suggested (10). The timing of the main meal by itself seems to be the most determinant factor in weight loss effectiveness, and therefore eating at the right time may be a relevant factor to consider in weight loss therapies.

The fact that it was the timing of lunch which related to the differences in weight loss effectiveness and not the timing of breakfast or dinner seems to be unexpected. However, it is important to note that in this Mediterranean population lunch is the biggest meal of the day and comprises approximately $40 \%$ of the total daily calories. This important intake of 
energy could be resetting peripheral clocks by itself or indirectly through changes in timing of the others meals. Indeed, the lunch timing was significantly correlated with the timing other meals in the current study. That the timing of food intake can influence the circadian system has been widely demonstrated (19), and may explain the lower insulin sensitivity as estimated by HOMA of late eaters as compared to early eaters (51).

Moreover, in this Mediterranean population the energy intake from breakfast was relatively low. So late lunch may prolong a "semi-fasting" state and induce glucose metabolism impairments. On the other hand, the energy intake from lunch was approximately 3 times larger than that from breakfast which may have been sufficient to maintain adequate glucose metabolism, even if dinner was late.

On the other hand, differences in insulin sensitivity by itself may have influenced food timing, because insulin resistance has been related to a delayed gastric emptying and impaired colonic transit time (52).

The current study explores the topic of timing in weight loss programs. One strength is that the sample is relatively large with numerous exposure variables including biomarkers and genetic indicators. However, we must highlight that the current work is an observational study. Therefore, although the association obtained between timing of main meal and weight loss is a novel and important observation; further interventional studies should be performed in order to demonstrate the causality of this observation.

In summary, eating late may impair the success of weight loss therapy. Unexpectedly, total energy intake, dietary composition, and estimated energy expenditure were not explaining these results. Changes in the chronotype, genetic background, and/or circadian system function may be implicated in this outcome. The current findings may help in the development of novel therapeutic strategies incorporating not only the caloric intake and macronutrient distribution — as is classically done-but also the timing of food.

\section{Acknowledgments}

Sources of support: This study was supported by grants from Tomás Pascual and Pilar Gómez-Cuétara Foundations, Spanish Government of Science and Innovation (BFU2011-24720), Séneca Foundation from the Government of Murcia (15123/PI/10). National Heart, Lung, and Blood Institute grants HL-54776, National Institute of Diabetes and Digestive and Kidney Diseases, Grant Number DK075030 and by contracts 53-K06-5-10 and 58-1950-9-001 from the US Department of Agriculture Research, and by National Heart, Lung, and Blood Institute grant R01 HL094806, and by National Institute of Diabetes and Digestive and Kidney Diseases, grant R21 DK089378.

\section{References}

1. Corbalán MD, Morales EM, Canteras M, Espallardo A, Hernández T, Garaulet M. Effectiveness of cognitive-behavioral therapy based on the Mediterranean diet for the treatment of obesity. Nutrition. 2009; 25(7-8):861-9. [PubMed: 19539176]

2. Teixeira PJ, Silva MN, Coutinho SR, Palmeira AL, Mata J, Vieira PN, et al. Mediators of weight loss and weight loss maintenance in middle-aged women. Obesity (Silver Spring). 2010; 18(4):72535. [PubMed: 19696752]

3. Garaulet M, Sánchez-Moreno C, Smith CE, Lee YC, Nicolás F, Ordovás JM. Ghrelin, sleep reduction and evening preference: relationships to CLOCK 3111 T/C SNP and weight loss. PLoS One. 2011; 6(2):e17435. [PubMed: 21386998]

4. Garaulet M, Esteban Tardido A, Lee YC, Smith CE, Parnell LD, et al. SIRT1 and CLOCK $3111 \mathrm{~T}>\mathrm{C}$ combined genotype is associated with evening preference and weight loss resistance in a behavioral therapy treatment for obesity. Int J Obes (Lond). 2012 (in press). 
5. Garaulet M, Corbalán-Tutau MD, Madrid JA, Baraza JC, Parnell LD, Lee YC, et al. PERIOD2 variants are associated with abdominal obesity, psycho-behavioral factors, and attrition in the dietary treatment of obesity. J Am Diet Assoc. 2010; 110(6):917-21. [PubMed: 20497782]

6. Oishi K, Shirai H, Ishida N. CLOCK is involved in the circadian transactivation of peroxisomeproliferator-activated receptor alpha (PPARalpha) in mice. Biochem J. 2005; 386:575-581. [PubMed: 15500444]

7. Turek FW, Joshu C, Kohsaka A, Lin E, Ivanova G, McDearmon E, et al. Obesity and metabolic syndrome in circadian Clock mutant mice. Science. 2005; 308:1043-1045. [PubMed: 15845877]

8. Marcheva B, Ramsey KM, Buhr ED, Kobayashi Y, Su H, Ko CH, et al. Disruption of the clock components CLOCK and BMAL1 leads to hypoinsulinaemia and diabetes. Nature. 2010; 466:627631. [PubMed: 20562852]

9. Froy O. Metabolism and circadian rhythms--implications for obesity. Endocr Rev. 2010; 31:1-24. [PubMed: 19854863]

10. Arble DM, Bass J, Laposky AD, Vitaterna MH, Turek FW. Circadian timing of food intake contributes to weight gain. Obesity (Silver Spring). 2009; 17:2100-2102. [PubMed: 19730426]

11. Sherman H, Genzer Y, Cohen R, Chapnik N, Madar Z, Froy O. Timed high-fat diet resets circadian metabolism and prevents obesity. FASEB J. 2012 (in press).

12. Lowrey PL, Takahashi JS. Mammalian circadian biology: elucidating genome-wide levels of temporal organization. Annu Rev Genomics Hum Genet. 2004; 5:407-41. [PubMed: 15485355]

13. Scheer FA, Hilton MF, Mantzoros CS, Shea SA. Adverse metabolic and cardiovascular consequences of circadian misalignment. Proc Natl Acad Sci U S A. 2009; 106(11):4453-8. [PubMed: 19255424]

14. Gómez-Abellán P, Madrid JA, Ordovás JM, Garaulet M. Chronobiological aspects of obesity and metabolic syndrome. Endocrinol Nutr. 2012; 59(1):50-61. [Article in Spanish]. [PubMed: 22100640]

15. Garaulet M, Ordovás JM, Gómez-Abellán P, Martínez JA, Madrid JA. An approximation to the temporal order in endogenous circadian rhythms of genes implicated in human adipose tissue metabolism. J Cell Physiol. 2011; 226(8):2075-80. [PubMed: 21520059]

16. Loboda A, Kraft WK, Fine B, Joseph J, Nebozhyn M, Zhang C, et al. Diurnal variation of the human adipose transcriptome and the link to metabolic disease. BMC Med Genomics. 2009; 2:7. [PubMed: 19203388]

17. Ptitsyn AA, Zvonic S, Conrad SA, Scott LK, Mynatt RL, Gimble JM. Circadian clocks are resounding in peripheral tissues. PLoS Comput Biol. 2006; 2(3):e16. [PubMed: 16532060]

18. Tu BP, Kudlicki A, Rowicka M, McKnight SL. Logic of the yeast metabolic cycle: temporal compartmentalization of cellular processes. Science. 2005; 310(5751):1152-8. [PubMed: 16254148]

19. Garaulet M, Madrid JA. Chronobiological aspects of nutrition, metabolic syndrome and obesity. Adv Drug Deliv Rev. 2010; 62(9-10):967-78. [PubMed: 20580916]

20. Garaulet M, Corbalán MD, Madrid JA, Morales E, Baraza JC, Lee YC, et al. CLOCK gene is implicated in weight reduction in obese patients participating in a dietary programme based on the Mediterranean diet. Int J Obes (Lond). 2010; 34(3):516-23. [PubMed: 20065968]

21. Garaulet M, Pérez-Llamas F, Zamora S, Tebar FJ. Weight loss and possible reasons for dropping out of a dietary/behavioural programme in the treatment of overweight patients. J Human Nutr Diet. 1999; 12:219-227.

22. Serra-Majem L, Aranceta J. SENC Working Group on Nutritional Objectives for the Spanish Population. Spanish Society of Community Nutrition. Nutritional objectives for the Spanish population. Consensus from the Spanish Society of Community Nutrition. Public Health Nutr. 2001; 4(6A):1409-13. [PubMed: 11918491]

23. Cooper, Z.; Fairburn, CG.; Hawker, DM. A clinician's guide. New York: The Guilford Press; 2003. Cognitive-Behavioral treatment of obesity.

24. Ferrario VF, Sforza C, Schmitz JH, Miani A Jr, Taroni G. Fourier analysis of human soft tissue facial shape: sex differences in normal adults. J Anat. 1995; 187(Pt 3):593-602. [PubMed: 8586558] 
25. Alberti KG, Eckel RH, Grundy SM, Zimmet PZ, Cleeman JI, Donato KA, Fruchart JC, James WP, Loria CM, Smith SC Jr. International Diabetes Federation Task Force on Epidemiology and Prevention; Hational Heart, Lung, and Blood Institute; American Heart Association; World Heart Federation; International Atherosclerosis. Society; International Association for the Study of Obesity. Harmonizing the metabolic syndrome: a joint interim statement of the International Diabetes Federation Task Force on Epidemiology and Prevention; National Heart, Lung, and Blood Institute; American Heart Association; World Heart Federation; International Atherosclerosis Society; and International Association for the Study of Obesity. Circulation. 2009; 120(16):1640-5. [PubMed: 19805654]

26. Pérez-Llamas F, Garaulet M, Herrero F, Palma JT, Pérez de Heredia F, et al. Multivalent informatics application for studies of the nutritional status of the population. Assessment of food intake. Nutr Hosp. 2004; 19(3):160-6. [Article in Spanish]. [PubMed: 15211725]

27. Mataix, J.; Mañas, M.; Llopis, J.; Martínez, E. [Table of composition of Spanish foods] Tabla de composición de alimentos españoles (in Spanish). Granada, Spain: Instituto de Nutrición y Tecnología, Universidad de Granada; 1995.

28. Moreiras, O.; Carvajal, A.; Cabrera, L. [Table of composition of Spanish foods] Tablas de composición de alimentos (in Spanish). Pirámide, SA., editor. Madrid: 1995.

29. Craig CL, Marshall AL, Sjöström M, Bauman AE, Booth ML, Ainsworth BE, Pratt M, Ekelund U, Yngve A, Sallis JF, Oja P. International Physical Activity Questionnaire: 12-country reliability and validity. Med Sci Sports Exerc. 2003; 35:1381-1395. [PubMed: 12900694]

30. Roman-Viñas B, Serra-Majem L, Hagstrom M, Ribas-Barba L, Sjostrom M, Segura-Cardona R. International Physical Activity Questionnaire: Reliability and validity in a Spanish population. European Journal of Sport Science. 2010; 10(5):297-304.

31. Horne JA, Ostberg O. A self-assessment questionnaire to determine morningness-eveningness in human circadian rhythms. Int J Chronobiol. 1976; 4(2):97-110. [PubMed: 1027738]

32. Taillard J, Philip P, Chastang JF, Bioulac B. Validation of Horne and Ostberg morningnesseveningness questionnaire in a middle-aged population of French workers. J Biol Rhythms. 2004; 19(1):76-86. [PubMed: 14964706]

33. Garaulet M, Lee YC, Shen J, Parnell LD, Arnett DK, Tsai MY, et al. CLOCK genetic variation and metabolic syndrome risk: modulation by monounsaturated fatty acids. Am J Clin Nutr. 2009; 90(6):1466-75. [PubMed: 19846548]

34. Garaulet M, Lee YC, Shen J, Parnell LD, Arnett DK, Tsai MY, et al. Genetic variants in human CLOCK associate with total energy intake and cytokine sleep factors in overweight subjects (GOLDN population). Eur J Hum Genet. 2010; 18(3):364-9. [PubMed: 19888304]

35. Sookoian S, Gemma C, Gianotti TF, Burgueño A, Castaño G, Pirola CJ. Genetic variants of Clock transcription factor are associated with individual susceptibility to obesity. Am J Clin Nutr. 2008; 87(6):1606-15. [PubMed: 18541547]

36. Jakubowicz D, Froy O, Wainstein J, Boaz M. Meal timing and composition influence ghrelin levels, appetite scores and weight loss maintenance in overweight and obese adults. Steroids. 2012; 77(4):323-31. [PubMed: 22178258]

37. DiTacchio L, Bushong EA, Gill S, Leblanc M, Chaix A, Joens M, et al. Time-restricted feeding without reducing caloric intake prevents metabolic diseases in mice fed a high-fat diet. Cell Metab. 2012; 15:848-60. [PubMed: 22608008]

38. Fonken LK, Workman JL, Walton JC, Weil ZM, Morris JS, Haim A, et al. Light at night increases body mass by shifting the time of food intake. Proc Natl Acad Sci U S A. 2010; 107(43):18664-9. [PubMed: 20937863]

39. Sensi S, Capani F. Chronobiological aspects of weight loss in obesity: effects of different meal timing regimens. Chronobiol Int. 1987; 4(2):251-61. [PubMed: 3508745]

40. Mistlberger RE, Lukman H, Nadeau BG. Circadian rhythms in the Zucker obese rat: assessment and intervention. Appetite. 1998; 30(3):255-67. [PubMed: 9632457]

41. Lowden A, Moreno C, Holmbäck U, Lennernäs M, Tucker P. Eating and shift work - effects on habits, metabolism and performance. Scand J Work Environ Health. 2010; 36(2):150-62.

[PubMed: 20143038] 
42. Ma Y, Bertone ER, Stanek EJ 3rd, Reed GW, Hebert JR, Cohen NL, et al. Association between eating patterns and obesity in a free-living US adult population. Am J Epidemiol. 2003; 158(1): 85-92. [PubMed: 12835290]

43. Colles SL, Dixon JB, O’Brien PE. Night eating syndrome and nocturnal snacking: association with obesity, binge eating and psychological distress. Int J Obes (Lond). 2007; 31:1722-1730. [PubMed: 17579633]

44. Corbalán-Tutau MD, Madrid JA, Garaulet M. Timing and duration of sleep and meals in obese and normal weight women. Association with increase blood pressure. Appetite. 2012; 59(1):9-16. [PubMed: 22450522]

45. Garaulet M, Ortega FB, Ruiz JR, Rey-López JP, Béghin L, Manios Y, et al. Short sleep duration is associated with increased obesity markers in European adolescents: effect of physical activity and dietary habits. The HELENA study. Int J Obes (Lond). 2011; 35(10):1308-17. [PubMed: 21792170]

46. Taheri S, Lin L, Austin D, Young T, Mignot E. Short sleep duration is associated with reduced leptin, elevated ghrelin, and increased body mass index. PLoS Med. 2004; 1(3):e62. [PubMed: 15602591]

47. Baron KG, Reid KJ, Kern AS, Zee PC. Role of sleep timing in caloric intake and BMI. Obesity (Silver Spring). 2011; 19(7):1374-81. [PubMed: 21527892]

48. Bienertová-Vasků J, Bienert P, Forejt M, Tomandl J, Brázdová Z, Vasků A. Genotype x nutrient association of common polymorphisms in obesity-related genes with food preferences and time structure of energy intake. Br J Nutr. 2010; 103(3):352-9. [PubMed: 19747414]

49. Corbalán-Tutau MD, Madrid JA, Ordovás JM, Smith CE, Nicolás F, Garaulet M. Differences in daily rhythms of wrist temperature between obese and normal-weight women: associations with metabolic syndrome features. Chronobiol Int. 2011; 28(5):425-33. [PubMed: 21721858]

50. Corbalán-Tutau D, Madrid JA, Nicolás F, Garaulet M. Daily profile in two circadian markers "melatonin and cortisol" and associations with metabolic syndrome components. Physiol Behav. 2012 (in press).

51. Zhao Y, Zhang Y, Zhou M, Wang S, Hua Z, Zhang J. Loss of mPer2 increases plasma insulin levels by enhanced glucose-stimulated insulin secretion and impaired insulin clearance in mice. FEBS Lett. 2012; 586(9):1306-11. [PubMed: 22504074]

52. Konturek PC, Brzozowski T, Konturek SJ. Gut clock: implication of circadian rhythms in the gastrointestinal tract. J Physiol Pharmacol. 2011; 62(2):139-50. [PubMed: 21673361]

53. Scheer FA, Morris CJ, Shea SA. The Internal Circadian Clock Increases Hunger and Appetite in the Evening Independent of Food Intake and Other Behaviors. Obesity (Silver Spring). 2013; 21:421-3. [PubMed: 23456944] 


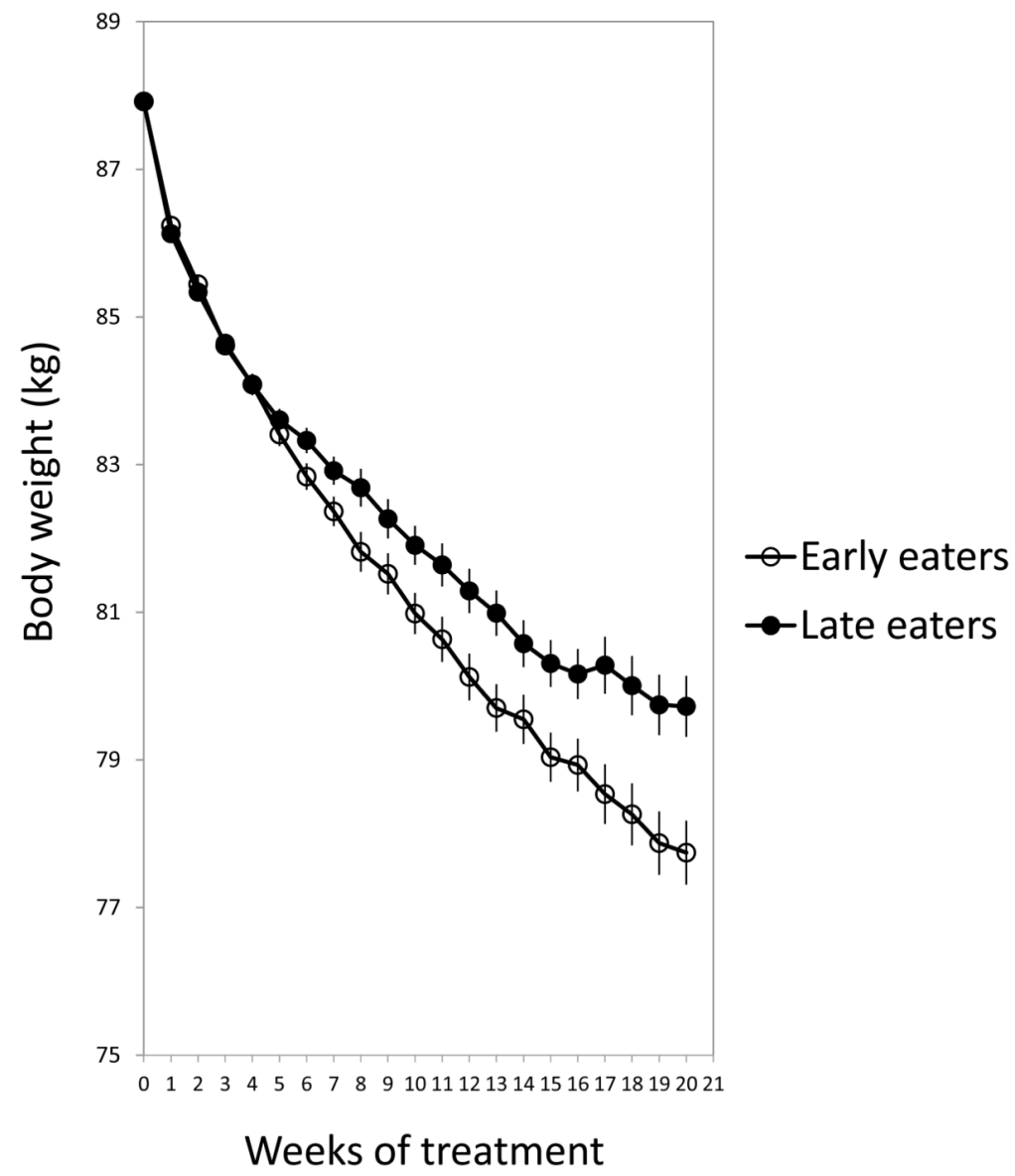

Figure 1.

represents the weight loss evolution of late and early lunch eaters during the 20 weeks of treatment. A repeated-measures analysis of variance (ANOVA) was performed. We adjusted analyses for sex, age center, and initial weight $(P=0.002)$. 


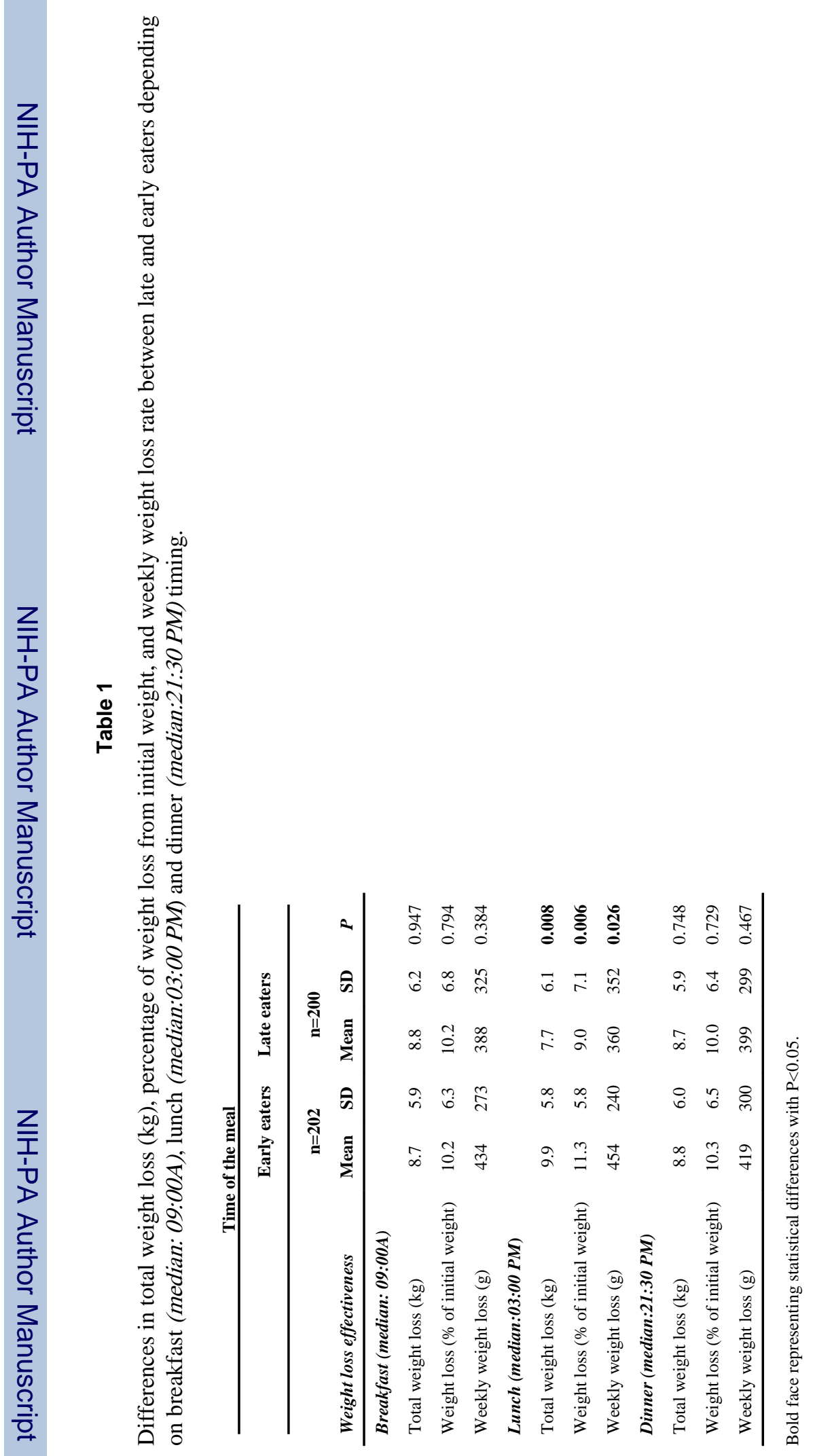




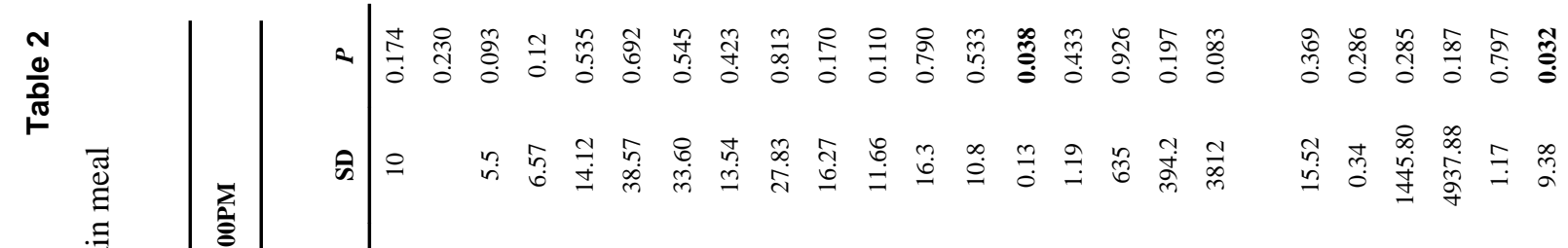

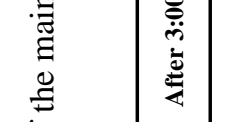

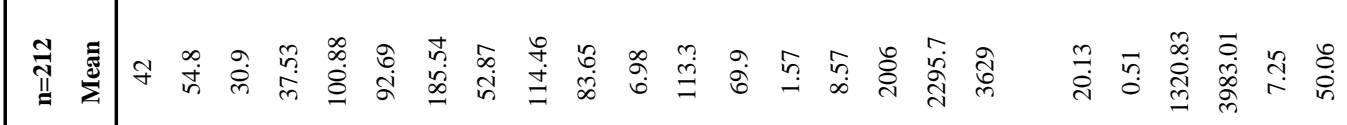

$$
\begin{aligned}
& \text { 范 }
\end{aligned}
$$

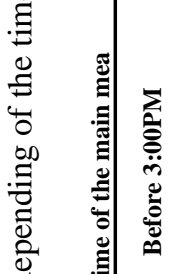

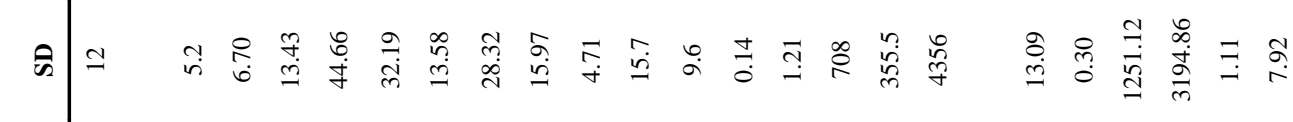

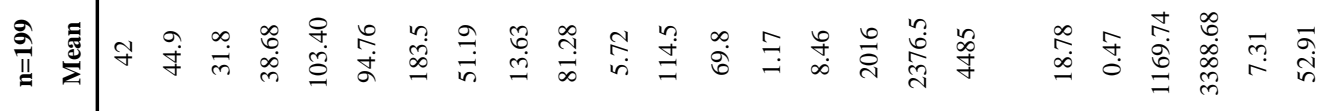

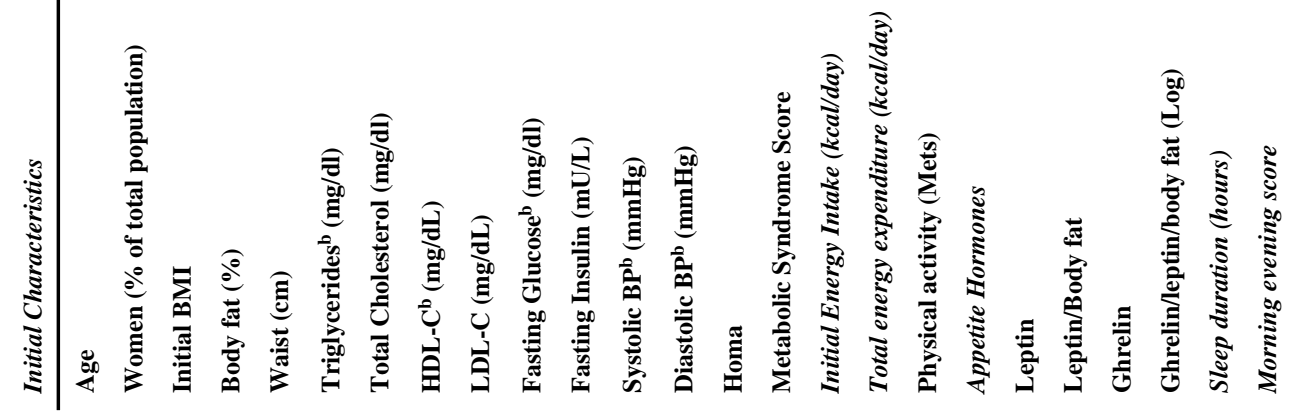




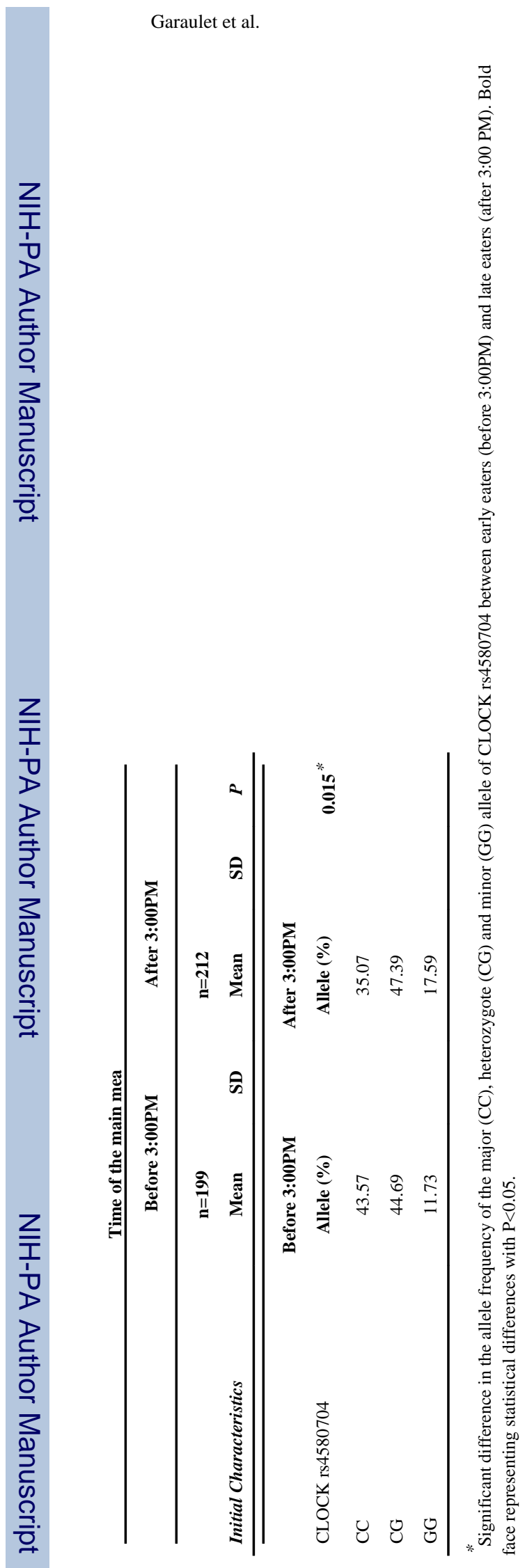

Int J Obes (Lond). Author manuscript; available in PMC 2013 October 01. 


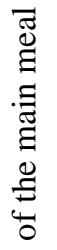




\section{Table 4}

Lack of differences in weight loss with sleep duration $(<$ median $7 \mathrm{~h}\rangle)$ morning evenningnes score $(<$ median score $\gg$ ) and genetic backgrounds (CLOCK rs4580704) (Minor and major).

\begin{tabular}{lrrc} 
& \multicolumn{3}{c}{ Weight loss (\% initial weight) } \\
\hline & Mean & SD & $\boldsymbol{P}$ \\
\hline Sleep (h) & & & \\
$<7 \mathrm{~h}$ & 9.50 & 6.93 & 0.747 \\
$\geq 7 \mathrm{~h}$ & 10.29 & 6.46 & \\
Morning/evening Questionnaire (score) & & & \\
$<53$ & 10.29 & 6.33 & 0.456 \\
253 & 10.17 & 6.57 & \\
CLOCK rs4580704 & & & \\
GG+CG & 10.05 & 6.56 & 0.644 \\
CC & 10.34 & 6.35 & \\
\hline
\end{tabular}

Int J Obes (Lond). Author manuscript; available in PMC 2013 October 01. 